\title{
Interactive Grouping of Friends in OSN: Towards Online Context Management
}

\author{
Bo Gao*, Bettina Berendt*, Dave Clarke*, Ralf De Wolf ${ }^{\dagger}$, Thomas Peetz*, Jo Pierson ${ }^{\dagger}$, and Rula Sayaf* \\ * Department of Computer Science \\ Katholieke Universiteit Leuven, \\ Email: firstname.lastname@cs.kuleuven.be \\ $\dagger$ †MIT - Studies on Media Information and Telecommunication \\ Vrije Universiteit Brussel, \\ Emails: ralf.de.wolf@vub.ac.be, jo.pierson@vub.ac.be
}

In: 2012 IEEE 12Th International Conference on Data Mining Workshops (PP. 555-562). IEEE Computer SCIEnCE Press, 2012.

\begin{abstract}
In Online Social Networks (OSNs), it can be difficult to maintain the context of a conversation or action, i.e. to know what the situation is and how to act appropriately. The resulting uncertainties may lead to privacy issues. We focus on one issue Context Collision in this paper, and motivate that a first step to address this issue is to help users distinguish groups of contacts within their OSN accounts. We conducted a small user study to investigate the criteria of users grouping the people they know. We summarized our participants' strategy of labeling the groups and found that they perform the grouping mainly by their connections with others. Informed by this study, we adopted a friend-graph-based community detection approach and developed an semi-automatic and interactive grouping tool FreeBu to aid in the grouping process.
\end{abstract}

Keywords-online social networks; privacy; transparency tool; context; visualization

\section{INTRODUCTION}

This paper introduces a software tool that helps users construct friend groups in OSNs. This section motivates our development of this tool.

Over recent years, we witness the tremendous growth of OSNs [6] such as Facebook (www.facebook.com) or Google+ (www.plus.google.com). More and more users participate in online social activities. According to Facebook ${ }^{1}$, there are 845 million users on Facebook and 483 million daily active users on average by the end of 2011. According to Lampe [19], the purpose of people using Facebook is primarily for maintaining their previous, offline relationships. As the PewInternet 2011 report $^{2}$ shows: an individual has met $89 \%$ of her Facebook friends more than once offline. Research has indicated that, by publishing (personal) information, OSN users are engaged in Impression Management [25], [14], [28] and building Social Capital [12]. Impression Management refers to the process in which people attempt to influence the perceptions of other people about a person, typically, this person is the user herself. Social Capital refers to the resources accumulated through the relationships among people [9].

\footnotetext{
${ }^{1}$ http://newsroom.fb.com/ [Accessed on Jun 4, 2012]

${ }^{2}$ http://goo.gl/xmAeG [Accessed on Mar 18, 2012]
}

Given its enormous user base, publishing nature and close connection to the offline world, OSNs often face privacy issues. One widely discussed phenomenon is Context Collision or Context Collapse [5], [20], [23]. As "context" is a multifaceted concept [11], [15], due to the scope of this paper, we do not intend to articulate a comprehensive definition for "context". However, studies have identified the following two relevant aspects: first, a context contains a group of people, and a particular role is expected from the person when she is within this context, to which we refer as the role-playing aspect [24]; second, the people within a context "are closely related to each other, in such a way that one would expect information about the user's interactions with one of them to become known to the others" [10], to which we refer as the information-enclosing aspect. Therefore, the information exchange among the members of the same context is usually more private than that among the members from different contexts. For example, a person has two contexts, one is at a company where he is an employee, the other is at home, where he is a husband. He plays two different roles within two different groups of people. The conversations between him and his family is private to his colleagues at work and vice versa. Context Collision refers to the phenomenon that the boundaries among people are blurred, the contexts in which they reside are mixed.

In an offline environment it is not difficult to distinguish between different contexts, because most of the time we know what topics we can talk about, or how we should behave toward others within a specific group of people. Whereas in an online environment, due to the lack of contextualization mechanism in OSNs (as discussed in Section II), such discernment is weakened. Context Collision makes it difficult for a user to control the flow of her personal information in OSNs. Following the theoretical framework of Goffman [13], De Wolf and Pierson point out that there is a lack of alternation between the front and back stages in OSNs. This alternation is necessary in constructing a personal identity. But Context Collision deprives the user's ability to "act on stage" [26].

If not taking into account the internal demarcation options within OSNs (such as Google+ circles), a user often behaves 
in the following two ways without the clear boundaries between contexts. One is to simply address a specific group of friends while others also see the published information. Alternatively, the user applies the lowest denominator strategy [18] - to only post the information that is suitable for all of her friends. In the former, privacy suffers; in the latter, the user's possibilities to realize different identities are severely constrained.

We see two solution approaches to help the user control her online information flow: 1) Find new ways in controlling the information flow, without the necessity of the user to place her friends into a certain role; or 2) Focus on facilitating the existing demarcation process through privacy feedback and awareness (PFA) tools that are more usercentered. In this paper we choose the latter option.

A first step towards online context management is to help the user distinguish groups of friends in OSNs. PFA tools provide the user with appropriate grouping recommendation as feedback. Once the user is aware of the grouping of her friends in OSNs, it becomes easier for her to decide what to post to whom. Berendt [2] examined the PFA approach regarding information literacy and privacy - the idea of PFA tools is to "show users within the context of their potentially privacy-related activities (e.g. within their social network platforms) important consequences of activities they have performed". Acquisti et al. [16] introduced the concept of "nudging" or "soft paternalism" towards privacy. Also, people are inherently curious about their online social data [4]. PFA tools can foster users' understanding and reflection of their privacy. The interactive grouping tool we introduce in this paper is a PFA tool.

The contributions of this paper are: First, we conduct a user study to investigate the criteria what users use to group their friends and other people they know. This provides us a basis to generate descriptions (labels) of detected communities in the tool. Second, based on the findings from the user study, we motivate our choice of graph-based community detection algorithm. Third, we describe a method and an interactive tool for community detection (Section IV-B1) and labeling (Section IV-B2).

\section{RELATED WORK}

Facebook and Google+ have developed grouping features. Users can create friend lists or circles to distinguish their friends. We identify three limitations of the current grouping approaches in Facebook and Google+:

1. Lack of automated process. Users need to manually construct friend groups such as Facebook lists or Google+ circles, which takes time. The only exception is that Facebook has offered users the automatic grouping function "smartlist". Four types of attributes are taken into account as the grouping criteria and four corresponding smartlists are generated, namely work, school, family and city. De Wolf and Pierson [27] found that sometimes users think the smartlists are too large, not correct or not relevant, and it appears that people have different criteria in delineating their contexts.

This restricted, attribute-based grouping is also unable to recognize different names of one institution. For example, people from the same school may fill in the name of their school differently, some use abbreviations, some use full name, etc.. This could result in more than one smart list generated about the same school. Smartlist is also limited by the sparsity of the data, i.e. people often do not fill in the information about work, school, family or city.

2. Lack of hierarchy. It is one layer of grouping. The user may want to subdivide a group to make a more fine-grained distinction.

3. Lack of visual presentation. Facebook adopts its traditional web form, the user needs to click a certain list to see who are the group members, how many people inside. Google+ has a more advanced interface where the user can intuitively drag and drop people into different circles. The name and the number of people are displayed on top of a circle. When the mouse hovers over a circle, the photos of the people in this circle are displayed. We can improve this kind of interface by adding more visual cues and interactions. See Section IV-C.

Other tools or proposed solutions that target OSN users with grouping features include: PViz [21] is a privacy-policy comprehension tool that shows the user the visibility of her profile according to the grouping of friends. Social $\mathrm{Graph}^{3}$ is a Facebook application that detects communities among user's friends based on the friend graph [3]. Meurs Challenger ${ }^{4}$ is another Facebook application similar to Social Graph, although not mentioned in the application description, its underlying clustering algorithm seems to be also based on graphs ${ }^{5}$. InMaps visualizes user's network on LinkedIn (www.linkedin.com), which is inspired by Gephi (www.gephi.org). Gephi is a general-purpose network visualization tool that can layout and color-code clustering structures of networks. Bacon and Dewan proposed a friend list recommendation algorithm based on friend cliques [1].

We find that these studies adopt a graph-based community detection approach, with various methods. But in the published materials that describe the corresponding implementations, none directly motivates the reason why such approach is adopted. Bacon and Dewan [1] mention that the friends' detailed information is largely not filled out by the users and it is possible that smart list generates duplicative lists as discussed in the first point. However, to detect communities as recommendations among a user's friends using their connections is only based on the assumption that it would be useful to the user. In this paper, we present a

\footnotetext{
${ }^{3}$ http://goo.gl/zw2d2 [Accessed on Apr 10, 2012]

${ }^{4} \mathrm{http}: / / g o o . g l / j z o 8 G$ [Accessed on Apr 10, 2012]

${ }^{5}$ Object graph obtained from Facebook API, such as friend graph, location graph, movie graph, etc..
} 
user study that solidifies this assumption. Note that we use the word "community" interchangeably with "context" as defined in Section I.

It also appears that few have provided an informative graphical user interface to the user with the meta-information about the detected communities. Meta-information is the information about the properties or characteristics of the formed communities. A user can be informed of such information by various visual cues, including textual labels, which only Pviz has applied to its user interface. But, full automation of friend community recommendation is not advisable since a user may group her friends based on a variety of reasons, it is important to leave room for manual and interactive adjustment by the user.

\section{USER STUDY}

\section{A. Background}

In order to provide sensible grouping recommendations to a user, we need to understand the criteria users apply to grouping people. As social networking sites are structured as personal networks [6], usually one OSN account corresponds to one individual, and the individual, as the center of the network, interacts with her friends online. Hence, we base our approach on an egocentric perspective.

In the user study, we asked a participant to group the people she knows personally. We refer to each person as $E$, as in "Ego", and the people $E$ knows personally are denoted as $F(E)$. Note that $F(E)$ may include both online and offline contacts of $E$, since $E$ 's OSN contacts do not cover the whole set of $E$ 's contacts in her life. By including offline contacts, we encourage $E$ to think independently of OSNs, to reflect upon what is essential for her to create boundaries/ contexts via such grouping, in the hope of discovering a grouping structure that is not distorted due to the limitations of online platforms.

However, one common question asked before any of the groupings to be performed is: "why do we want to group the people we know?", or "what is the purpose of the grouping?". It may appear to a user that, without any particular purpose defined, it is not sensible (or even possible) for her to create such grouping. Some argue that different purposes yield very different grouping structures.

While forming contexts is necessary to protect the user's privacy and help the user present herself appropriately online, the purposes of the formed contexts can be different. For example, a user wants to form a book club, he might group the people he knows into two categories, solely based on a book, one in which people like the book and the other not. The rest of the attributes of the people simply do not matter to the user in this purpose of grouping. We call this kind of grouping the specific-purpose grouping, which only rises to a specific occasion and only considers one or a few specific attribute(s).
There is another type of grouping, which is created when we don't bear any particular purpose in mind. Instead, we take a holistic view towards the people we know and make general divisions among them. We call it generalpurpose grouping. Such grouping can provide an immediate impression of $F(E)$ that is considered clear and sensible, so as to well illustrate and summarize the connections or relationships of $E$. Also because of its generality, $E$ can almost always construct other specific-purpose groupings by starting from the general-purpose one.

\section{B. Participants and Method}

We asked 15 participants to group the people they know in their lives. Out of the fifteen, six are $\mathrm{PhD}$ candidates in Computer Science, three are employees from different companies, the other six are Bachelor students from different universities. $40 \%$ of the participants are females. The ages range from twenty-two to thirty-one. Note that our selection of the users is mainly limited in terms of age. Nonetheless, given the fact that young people are driving the usage of Facebook [17], we believe this study holds value in aiding group-building in OSNs.

Each $E$ is asked to draw a grouping structure of $F(E)$, in a star-tree form. If $E$ is not familiar with the concept of star tree, an example is given, as in Figure 1. The participants were asked to construct the drawing on a computer with a drawing tool of their choice or on paper. It is an intuitive means that allows us to quickly identify $E$ 's grouping criteria. We give the following guidelines:

- Group the people in $E$ 's life, who $E$ knows personally and is alive.

- $E$ is supposed to be in the middle as the "self" in the example from which all the curves (i.e. branches) sprout.

- Hierarchy is allowed, i.e. a branch can split into more branches, each branch is considered as a group, the subbranch as the subgroup, and so on.

- The label on each branch characterizes or summarizes the people within that branch; if a branch contains only one person, a label may not be required.

- The same person can appear in different (sub) branches.

- Proceed with the grouping until that adding more people would not require new (sub) branch(es).

- Labeling the tips of the branches with names is encouraged, as illustrated in Figure 1, but not mandatory.

\section{Results and Interpretation}

We analyzed the fifteen general-purpose groupings of the participants by categorizing their labels and counting the frequencies of the labels. We observed that the grouping labels used were similar and categorized them into ten categories: Interests/ Hobbies (I/H), Education-related (edu), 


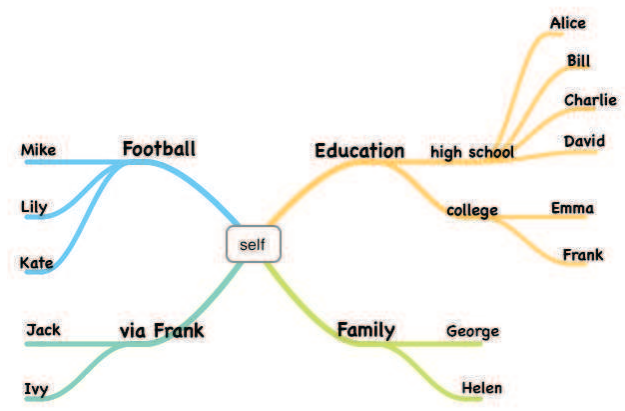

Figure 1. A grouping-tree example.

Work-related (work), Other-Organization-related (OO), Language/Nationalities (L/N), Location-related (loc), Timerelated (time), Age-related (age), Family-related (family) and Connection-related (conn).

Interests/ Hobbies labels indicate a group of people performing recurrent activities based on common interests, including keywords such as "skiing", "rugby", "concert group", "travel buddies", "pingpong club", etc.. Education-related labels include school, university and major names, degree titles, or the keywords such as "classmates", "fellow students", "professors", etc.. Workrelated labels mainly cover company names, project names, and the keywords such as "work", "leaders", "colleagues", etc.. Other-Organization-related labels indicate that people being grouped based on organizations that are different from schools, universities and corporations, e.g. a youth-movement organization. The labels of Language/Nationalities are used to distinguish groups of people with different nationalities, languages or ethnic backgrounds. Location-related labels categorize people based on locations, the keywords are city names. Time-related labels specifically indicate a period of time, such as "current", "old", "childhood". Age-related labels include "elderly", "peers", "senior", "junior", etc.. Family-related labels cover keywords such as "relatives", "(not) related-by-blood", "parents", etc.. Connection-related labels indicate the strengths or types of connections that $E$ has with other people. The keywords include "close", "best", "(dis)like", "acquainted", "not interested", etc., or secondary connections that express $E$ knowing a group of people via a specific person $X$, who acts as a bridge and is considered an important grouping indicator, the keywords of secondary connections include: " $X$ 's friends", " $X$ 's connections", "via $X$ ", etc..

To measure the importance of each category of the labels, we count the frequency of the labels in each category. The initial counting result is shown in Table I. The columns are the categories of the labels, the rows are Es. Note that some people perform the grouping in a more detailed way than others, i.e. with more labels. For example, if $E$ happens
Table I

LABEL COUNTS OF DIFFERENT CATEGORIES FOR EACH $E$

\begin{tabular}{|l|r|r|r|r|r|r|r|r|r|r|r|}
\hline & I/H & edu & work & OO & L/N & loc & time & age & family & conn \\
\hline E1 & 1 & 8 & 1 & 12 & 0 & 0 & 2 & 2 & 1 & 5 \\
\hline E2 & 0 & 4 & 1 & 0 & 2 & 0 & 0 & 0 & 4 & 3 \\
\hline E3 & 0 & 2 & 1 & 0 & 0 & 0 & 0 & 0 & 9 & 7 \\
\hline E4 & 3 & 6 & 1 & 1 & 0 & 0 & 0 & 0 & 1 & 44 \\
\hline E5 & 10 & 6 & 7 & 0 & 4 & 4 & 0 & 4 & 5 & 6 \\
\hline E6 & 2 & 3 & 4 & 0 & 1 & 1 & 0 & 0 & 1 & 4 \\
\hline E7 & 3 & 11 & 7 & 1 & 0 & 0 & 0 & 0 & 4 & 5 \\
\hline E8 & 0 & 0 & 1 & 0 & 0 & 0 & 0 & 0 & 3 & 2 \\
\hline E9 & 0 & 8 & 0 & 0 & 0 & 0 & 0 & 4 & 3 & 15 \\
\hline E10 & 0 & 6 & 0 & 0 & 0 & 0 & 0 & 4 & 3 & 8 \\
\hline E11 & 0 & 5 & 0 & 0 & 0 & 0 & 0 & 0 & 3 & 6 \\
\hline E12 & 0 & 4 & 1 & 0 & 0 & 1 & 0 & 1 & 4 & 12 \\
\hline E13 & 0 & 14 & 0 & 0 & 0 & 0 & 0 & 0 & 3 & 1 \\
\hline E14 & 2 & 0 & 0 & 0 & 0 & 0 & 0 & 0 & 1 & 15 \\
\hline E15 & 1 & 6 & 3 & 0 & 2 & 0 & 0 & 0 & 4 & 7 \\
\hline
\end{tabular}

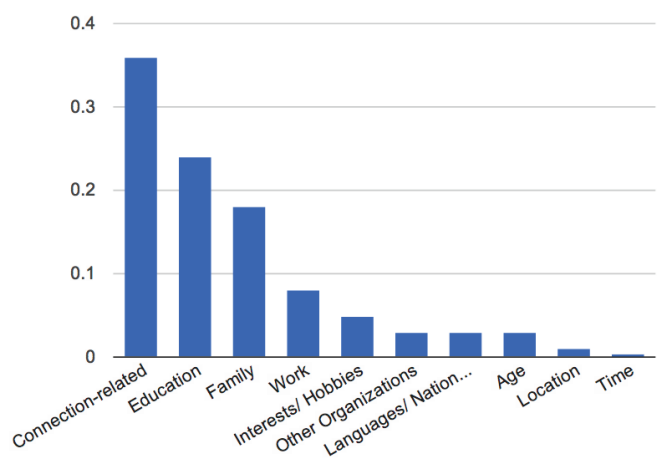

Figure 2. The Ranking of the Categories of Labels

to have a big family with various relatives, such situation may force her to put more family-related labels into the star tree, which gives us the impression that she emphasizes the importance of the family-related labels, while she actually considers the other categories of labels just as important.

To compare all the counts on the same scale, each E's counts are divided by her total number of counts respectively, deriving percentages. Then, we calculate the average percentage for each category of labels, the result is shown in Figure 2. On average, each $E$ uses $36 \%$ connection labels, $24 \%$ education labels, $18 \%$ family labels, $8 \%$ work labels.

We see that $E$ s perform the groupings primarily based on their connections with $F(E)$, they consider the types and strengths of these connections important. For example, from $E$ s' drawings, we observed that close friends and acquaintances were often prominently distinguished, or, a group of people who $E$ got to know through a friend was often emphasized, preceding other criteria. Also, the connectionrelated criterion and others often work in a mixed fashion. We observed that some $E$ s first grouped $F(E)$ according to schools, then within each school, people were divided based on connections, e.g. closed ones and acquainted ones. 
The observation on the mixed grouping strategy suggests that other important criteria such as education, family and work are inherently connection-based. Because people are more likely to form connections in the same family, school or workplace than in the different, and given the popularity of the groupings based on secondary connections, it follows that - during a grouping, $E$ considers not only her connections with $F(E)$, but also the connections among $F(E)$. These connections play a hidden but key role in the groupings with the non-connection-related criteria.

\section{Implications of the Results for Tool Design}

Essentially, the connection information is more informative than the attributes. While the attributes convey simple facts - education, family, work, hobbies, languages, etc. about people, the connection information may imply more complex relationships that are formed and shaped through a history of interpersonal interactions, and cannot be easily expressed by the attributes. Thus, the connection-based grouping is favored by the participants.

Recall the two aspects of a context from Section I, we see that during grouping, the participants consider both of the role-playing aspect (as in the attribute-based criteria) and the information-enclosing aspect (as in the connection-based criteria). The groups constructed in the general-purpose grouping are a manifestation of the privacy contexts of the participants.

Note that the user study is not an evaluation. Also because the small number of participants, the study can hardly be statistically relevant. But it serves as a useful starting point for us to inform the design choices of our grouping tool. More specifically, we select a user's Facebook data according to the grouping criteria for label derivation and adopt a graph-based community detection algorithm. Finally, we use a hierarchical tree structure as the visual presentation of the groups. All is elaborated in Section IV.

\section{A FRIEND-GROUPING TOOL FOR OSNS}

This section describes the method that we use to recommend an initial grouping of the user's OSN friends, while enabling the user to explore and adapt this grouping according to her needs, and eventually publish the results onto her OSN account. Due to the prevalence of Facebook among today's OSNs, we have implemented this tool based on Facebook data. The method is however applicable for any other OSNs that provide data access to its social graph and profile data. We call our tool FreeBu, short for Friend tree Bubbles.

\section{A. Data}

We base our PFA tool on the data retrieved via Facebook graph $\mathrm{API}^{6}$ with the user's access token. We aid the user

${ }^{6}$ https://developers.facebook.com/docs/authentication/ [Accessed on May 29, 2012] to group her Facebook friends, by firstly recommending an initial grouping structure, assigning appropriate labels to the groups and then letting her further adjust the grouping (see the following sections on Computational Model and User Interface). The grouping is constructed based on the user's friend graph, in which each node is a friend of the user's, if two friends are also friends to each other, they are linked. Note that such links are unweighted. We generate labels for the groups with collected attribute-based data:

If the user has family members registered as Facebook users and the user has indicated them as family members on Facebook, such information can be retrieved, including the names of the family members and their types of relationships with the user, e.g. cousin, aunt. Education-related data includes a list of schools, each school has its name and type, e.g. high school or graduate school, with possibly more information such as the year and the concentrations if the user has filled in. Work-related data includes a list of work-objects, each object contains the name and the location of the employer, the position of the user, the starting and ending time of the job. Language-related data includes a list of names of the languages that user speaks. For hobbyrelated data, we collect the "likes" of a user, which may contain anything, from sports to TV shows, from a public figure to a book, etc..

It is important to let the user form sensible groups on their own, by providing options of available OSN data on a meta-level. For example, what data attributes are available on OSN, how are people distributed over these attributes. Eventually, let the user decide what data is most relevant and what grouping structure is closest to what she has in mind.

\section{B. Method}

The task of recommending groups to a user is two-fold. First, to detect communities in user's online social network, second is to derive labels for each community based on the data described in Section IV-A.

1) Community Detection: As motivated previously, we adopt a graph-based community detection algorithm - more specifically, the Louvain method [3] - to extract communities from the user's friend graph. It is a heuristic method that is based on modularity optimization. As stated by the authors, the method is shown to outperform all other known community detection method in terms of computation time, with reasonably good quality of the detected communities, which is characterized by modularity. Modularity measures the density of links inside communities as compared to links between communities [22]. An area with more mutually connected friends is more likely to be identified as a community. The Louvain method outputs flat communities.

2) Label Derivation: To support the exploration of the visualization, and help the user identify the characteristics of different groups, it is critical to derive informative labels 
for communities. The label of a group should highlight the attributes of the people in it. We adopt the F-measure to determine the labels for the communities. F-measure is a standard measure combining precision and recall (Equation $1)$. As the labeling experiments in [21] indicate, $F$-measure comes out as one of the best labeling measures for communities detected with the user's Facebook data - the labels with high $F$-measure scores are often considered suitable by the users.

$$
F \text {-measure }=2 \frac{\operatorname{Precision}(C, A) * \operatorname{Recall}(C, A)}{\operatorname{Precision}(C, A)+\operatorname{Recall}(C, A)}
$$

with

$$
\begin{gathered}
\operatorname{Precision}(C, A)=\frac{|C \cap A|}{|A|} \\
\operatorname{Recall}(C, A)=\frac{|C \cap A|}{|C|}
\end{gathered}
$$

$C$ denotes a set of people within the same community $c$, $A$ denotes a set of people with the same attribute-value $a$, e.g. certain name of a university. Precision $(C, A)$ measures the percentage of people with the attribute-value $a$ in the community $c$ to the whole population with attribute-value $a$. $\operatorname{Recall}(C, A)$ measures the percentage of people with the attribute-value $a$ in the community $c$ to the whole population of the community $c$.

For each community, a list of labels is generated based on all the data attributes mentioned in Section IV-A, and then sorted according to every label's $F$-measure score. The user can determine the number of labels appearing on the communities. The labels with higher $F$-measure scores are firstly selected.

\section{User Interface}

In this section, we describe the graphical user interface of the grouping tool, including its visual presentation and how the user can modify the grouping structure with the interface.

1) Visual Presentation: We adopt the star-tree form to represent the grouping structure. As shown in Figure 3, the nodes of the tree are represented by circles, each pair of parent-child nodes are connected by straight lines. The root of the tree (the blue circle in the middle) is the user herself, the red circles represent different communities detected by the algorithm, the leaves (the green circles surrounding the red ones) represent user's friends on Facebook. We scale the sizes of community circles based on the number of people within each community, a larger size corresponds to more people.

The labels are shown on top of the community circles, if a community contains more than one person. The user can click on one bubble - a community or a person - to zoom in to concentrate on a particular part of the tree. We blur the labels for privacy reasons. The labels are typically

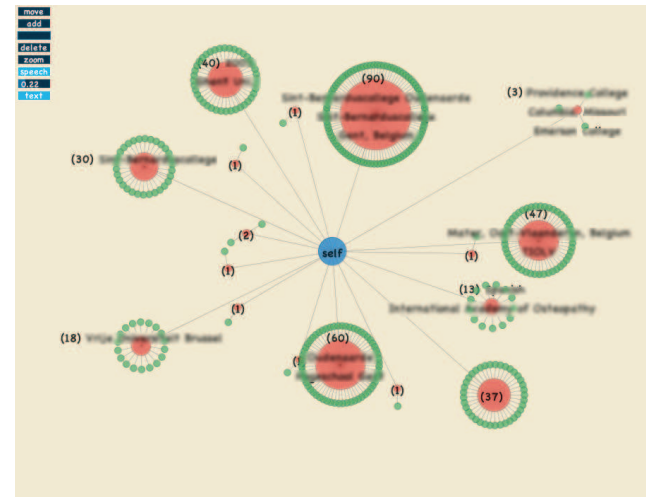

Figure 3. The overview of the user interface.

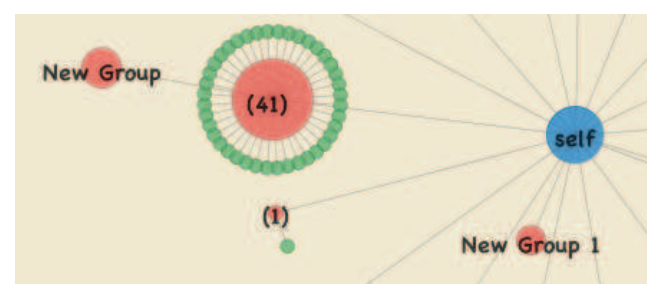

Figure 4. The user can add new groups at different levels of the star-tree, "New group" is added at level two, attached to the level-one circle labeled with " 41 ", "New group 1 " is added at level one, directly attached to the "self" circle. The user can also edit the labels of the circles.

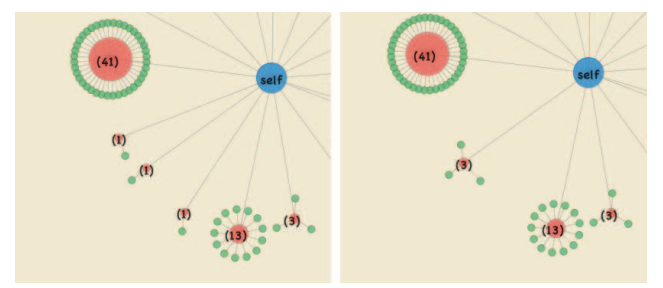

Figure 5. On the left, three individuals are initially assigned into three different groups; On the right, the user move the three individuals into one group.

school names, school years and work places. The number in front of the blurred labels indicate the number of people in the corresponding circles. The user can adjust the amount of labels shown by sliding the threshold bar.

2) Group Modification: Initially, we provide the user with one-layer grouping, the user can modify it by adding or removing (sub) groups, so that the user is able to construct her grouping hierarchically, as shown in Figure 4. The user can also change the members of the groups by "drag and drop" friends from one red circle to another, as shown in Figure 5.

\section{LimitaTiOnS AND FUtURE WORK}

\section{A. On the User Study}

The number of participants is small. The demographic structure of the sampled people in the user study is limited, 
mainly in terms of their age range, but also educational and cultural backgrounds. This can be improved by involving more and larger variety of people in the user study.

\section{B. On the Data}

We use friend graph data, in which a connection between two people is formed when the two are friends to each other. However, such friend graph data is not ideal to describe users' relationships, as we do not know the type and the strength of a connection in a given friend graph. A connection may be formed due to potentially any reason, e.g. that a pair of individuals have chatted pleasantly in an offline meeting and decide to become friends on Facebook, or two oldest and closest offline friends one day add each other on Facebook, or they have never met each other offline, but both are actively in an online forum, and then became Facebook friends.

As the friend graph lacks of detailed connection information, i.e. type and strength, it may cause deficiency when inputted to a community detection algorithm based on graph. For instance, someone $A$ in the user's friend graph is categorized into a community because $A$ has more links with the people in that community, meanwhile $A$ also connects to $B$ who is in another community. The user considers the linkage between $A$ and $B$ is much stronger than the rest of $A$ 's links to others, and $A$ should be put into $B$ 's community. The friend graph does not contain such knowledge. Further investigations may focus on the measurement of the strengths of connections and the involvement of the user's input, making the friend graph more informative.

Another limitation of the friend-graph data is that the user does not group her friends purely based on connections, sometimes on attributes as well. However, under what circumstances the user chooses attributes over connections to group friends seems rather random, and connection-based grouping is more generally applied by the user than attributebased according to our user study. In the next step, we can offer different versions of grouping recommendations to the user, including purely connection-based, purely attributebased grouping, and a mixture of both, so that the user is reminded with more alternatives and acquires more insights of the grouping structure of her friends.

One other limitation of the data holds in OSN in general the online OSN is not a perfect replicate of the offline world. Usually, critical data in an OSN is missing, or OSN is not synchronized or updated with the offline counterpart. Some important offline activities or events may not be shown on Facebook, a user may not fill in certain hobby on Facebook but indeed practice this hobby often and has a special group of friends, or most of a user's family members are easily not to be found on Facebook.

\section{On the Community Detection Algorithm}

The graph-based community detection algorithm derives communities by optimizing modularity. But modularity optimization is computationally hard [7]. Sometimes it is necessary to adopt approximation algorithms to deal with large graphs, as does the Louvain method.

Also, the Louvain method outputs a flat grouping structure for each given graph, while a hierarchical grouping structure may enhance the user's comprehension of her contexts.

As a person's social connections are complex in nature. There can be friends who bridge different groups. Algorithms that emphasize on discovering bridging/overlapping structures are potentially useful, as suggested in [8].

Moreover, as discussed in Section V-B, the connectionbased criteria for people grouping is favored by the participants. But it is not necessarily and not always the case, there are times that people do purely attribute-based grouping. To make more fine-tuned grouping recommendations, we should extend the community detection algorithm by taking into account attribute data on the nodes and more information on the edges, such as the types and weights of the edges, which can be derived from the commenting or the internal chatting messages of a user in OSN.

\section{On the Evaluation of the Tool}

Although the user study in [1] has suggested that connection-based friend group recommendation is favored over Facebook's smart-list, the star-tree form of the graphical presentation, the efficiency and the effectiveness of users' interactions with the interface are in need of evaluation. We plan to create tasks for users (to construct online friend groups), record, measure and compare the differences of their interactions with the grouping tool and with the Facebook list tool. We also plan to investigate how and to what extent that the grouping tool can aid users to enhance their privacy by creating contexts. Due to the complexity of these evaluations, we set them in our next step.

\section{SUMMARY}

In this paper, we have introduced an interactive grouping tool FreeBu using the user's Facebook data. We investigate the criteria users apply to grouping people, identify several data attributes that users frequently adopt for labeling their groups, and the inherently connection-based grouping method by users. We developed the grouping tool based on our findings in the user study that can help users recognize and modify the grouping of people. With the automatic community detection and labeling features in the tool, the user gains an overview of her friends on Facebook. According to this, the user can then adjust this grouping structure by adding, removing groups, rearranging the members inside the groups, and eventually apply the grouping decision onto her Facebook account. The grouping tool helps the user create sensible boundaries in OSN, which makes it easier for 
the user to decide what to publish or receive (sometimes very sensitive or personal) information to or from which group of people. We consider the interactive grouping tool as a first step to address the privacy concern Context Collision.

\section{ACKNOWLEDGMENT}

The research leading to these results has received funding from the Strategic Basic Research (SBO) Programme of the Flemish Agency for Innovation through Science and Technology (IWT) in the context of the SPION project (www.spion.me) under grant agreement number 100048 .

\section{REFERENCES}

[1] Kelli Bacon and Prasun Dewan. Towards automatic recommendation of friend lists. In Proc. CollaborateCom 2009, pages 1-5, Crystal City, Washington D.C., USA, 2009.

[2] Bettina Berendt. Data mining for information literacy. In D.E. Holmes and L.C. Jain, editors, Data Mining: Foundations and Intelligent Paradigms, volume 25, pages 265-297. Springer Berlin Heidelberg, Berlin, Germany, 2011.

[3] Vincent D Blondel, Jean-Loup Guillaume, Renaud Lambiotte, and Etienne Lefebvre. Fast unfolding of communities in large networks. Journal of Statistical Mechanics: Theory and Experiment, 2008(10):P10008 (12pp), 2008.

[4] danah m. boyd. Faceted id/entity: Managing representation in a digital world. Department of Media Arts and Sciences, Massachusetts Institute of Technology, 2002. http://smg. media.mit.edu/papers/danah/danahThesis.pdf [2012-08-08].

[5] danah m. boyd. Taken Out of Context: American Teen Sociality in Networked Publics. Social Science Research Network Working Paper Series, February 2009. http://www. danah.org/papers/TakenOutOfContext.pdf [2012-08-08].

[6] danah $\mathrm{m}$. boyd and Nicole B. Ellison. Social network sites: Definition, history, and scholarship. Journal of ComputerMediated Communication, 13(1):210-230, 2007.

[7] Ulrik Brandes, Daniel Delling, Marco Gaertler, Robert Grke, Martin Hoefer, Zoran Nikoloski, and Dorothea Wagner. On finding graph clusterings with maximum modularity. In Andreas Brandstädt, Dieter Kratsch, and Haiko Müller, editors, Graph-Theoretic Concepts in Computer Science, volume 4769 of Lecture Notes in Computer Science, pages 121-132. Springer, Berlin / Heidelberg, 2007.

[8] Remy Cazabet, Maud Leguistin, and Frederic Amblard. Automated community detection on social networks: useful? efficient? asking the users. In Proceedings of the 4th International Workshop on Web Intelligence \& Communities, New York, NY, USA, 2012. ACM.

[9] James S. Coleman. Social Capital in the Creation of Human Capital. American Journal of Sociology, 94:S95-S120, 1988.

[10] George Danezis. Inferring privacy policies for social networking services. In Proceedings of the 2nd ACM workshop on Security and artificial intelligence, AISec '09, pages 5-10, New York, NY, USA, 2009. ACM.

[11] Anind K. Dey. Understanding and using context. Personal and Ubiquitous Computing, 5(1):4-7, 2001.

[12] Judith Donath. Signals in social supernets. Journal of Computer-Mediated Communication, 13(1):231-251, 2007.

[13] Erving Goffman. The Presentation of Self in Everyday Life. Anchor Books, 1745 Broadway, New York, NY 10019 USA, 1959.
[14] S. D. Gosling, S. Gaddis, and S. Vazire. Personality impressions based on facebook profiles. In Proceedings of the International Conference on Weblogs and Social Media (ICWSM 2007), Boulder, Colorado 80302 USA, 2007. University of Maryland, Baltimore County. http://www.icwsm.org/papers/ 3--Gosling-Gaddis-Vazire.pdf [2012-08-10].

[15] Georg Groh. Contextual Social Networking. PhD thesis, University of Technology, Munich, 2012. Habilitation thesis in Computer Science.

[16] Ralph Gross, Alessandro Acquisti, and John Heinz. Information revelation and privacy in online social networks. In WPES '05: Proceedings of the 2005 ACM workshop on Privacy in the electronic society, pages 80,71 , Alexandria, VA, USA, 2005. ACM.

[17] Keith Hampton, Lauren S. Goulet, Lee Rainie, and Kristen Purcell. Social networking sites and our lives. Technical report, Pew Research Center's Internet \& American Life Project, June 2011.

[18] Bernie Hogan. The Presentation of Self in the Age of Social Media: Distinguishing Performances and Exhibitions Online. Bulletin of Science, Technology \& Society, 30(6):377-386, December 2010.

[19] Cliff Lampe, Nicole Ellison, and Charles Steinfield. A face(book) in the crowd: social searching vs. social browsing. In Proceedings of the 2006 20th anniversary conference on Computer supported cooperative work, CSCW 06 , pages 167-170, New York, NY, USA, 2006. ACM.

[20] Alice E. Marwick and danah m boyd. I tweet honestly, I tweet passionately: Twitter users, context collapse, and the imagined audience. New Media \& Society, 13(1):114-133, February 2011.

[21] K Mazzia, A; LeFevre and E. Adar. The PViz Comprehension Tool for Social Network Privacy Settings. Technical report, UM Tech Report CSE-TR-570-11, 2011. http://web.eecs.umich.edu/ klefevre/Publications_files/ CSE-TR-570-11.pdf [2012-08-08].

[22] M. E. J. Newman and M. Girvan. Finding and evaluating community structure in networks. Physical Review E, 69(2):026113.1-15, February 2004.

[23] Kate Raynes-Goldie. Aliases, creeping, and wall cleaning: Understanding privacy in the age of facebook. First Monday, 15(1), 2010. http://firstmonday.org/htbin/cgiwrap/bin/ojs/ index.php/fm/article/view/2775/2432 [2012-08-08].

[24] B. Schilit, N. Adams, and R. Want. Context-aware computing applications. In Proceedings of the 1994 First Workshop on Mobile Computing Systems and Applications, WMCSA '94, pages 85-90, Washington, DC, USA, 1994. IEEE Computer Society.

[25] Stephanie T. Tong, Brandon Van Der Heide, Lindsey Langwell, and Joseph B. Walther. Too Much of a Good Thing? The Relationship Between Number of Friends and Interpersonal Impressions on Facebook. Journal of Computer-Mediated Communication, 13(3):531-549, 2008.

[26] Ralf De Wolf, Rob Heyman, and Jo Pierson. Privacy by Design through social requirements analysis of social network sites from a user perspective. In Serge Gutwirth, Ronald Leenes, and Paul de Hert, editors, European Data Protection: Coming of Age. Springer, Berlin, Germany, 2012.

[27] Ralf De Wolf and Jo Pierson. Privacy beyond the individual: analysing group based access control models on social network sites from a user perspective. In IAMCR (International Association for Media and Communication Research), Durban, South-Africa, 2012.

[28] Shanyang Zhao, Sherri Grasmuck, and Jason Martin. Identity construction on facebook: Digital empowerment in anchored relationships. Computers in Human Behavior, 24(5):1816 1836, 2008. Including the Special Issue: Internet Empowerment. 